\title{
Control of Base-Isolated Systems using Force Feedback
}

\author{
Francesc Pozo, Arturo Rodríguez, Leonardo Acho, Yolanda Vidal and José Rodellar
}

\begin{abstract}
The combination of passive and active schemes has been increasingly considered in the structural control community as a promising way to design efficient smart hybrid base isolation systems for seismic protection. This paper considers a hybrid system in which an active feedback control law is derived to be applied in parallel with a passive isolation device. The active control uses the restoring force supplied by the passive isolator as the main feedback signal. This paper can be divided in two main parts: in the first one, the paper presents the theoretical formulation and stability analysis in the active control strategy; in the second part, a set of numerical simulations is performed when the force is supplied in a semiactive way to validate and discuss the efficiency of the approach in a more realistic scenario. Moreover, the performance of the proposed semi-active control algorithm is compared with passive-off, passive-on and clipped-optimal controllers. The proposed control scheme reduces the base displacement without increasing the floor accelerations.
\end{abstract}

\section{INTRODUCTION}

Structural control systems have shown great interest in the last decades for hazard mitigation in civil structures [9]. Passive control systems have been widely used to mitigate vibrations due to external dynamic loadings [15], [22]. The basic concept of base isolation is to make the structure behave like a rigid body through a certain degree of decoupling from the ground motion. However, for the purpose of maintaining the seismic response of structures within safety, service and comfort limits, the combination of passive base isolators and feedback controllers (applying forces to the base) has been proposed in recent years. Applications of hybrid control systems consisting of active ([4], [16], [17], [18]) or semi-active ([3], [7], [14]) systems installed in parallel to base isolators have the capability of reducing response quantities of base-isolated structures more significantly than passive dampers. The idea of adding a feedback control is based on the premise that a control action is to be applied at the base with force magnitudes which are not excessive due to the high flexibility of the isolators. The main benefit of the inclusion of the control

This work was supported by CICYT (Spanish Ministry of Science and Innovation) through grants DPI2008-06463-C02-01 and DPI2008-06564C02-02.

F. Pozo, L.Acho and Y. Vidal are with CoDAlab (codalab.ma3.upc.edu), Departament de Matemàtica Aplicada III, Escola Universitària d'Enginyeria Tècnica Industrial de Barcelona (EUETIB), Universitat Politècnica de Catalunya BarcelonaTECH (UPC), Comte d'Urgell, 187, 08036 Barcelona, Spain francesc.pozo@upc.edu, leonardo.acho@upc.edu, yolanda.vidal@upc.edu

A. Rodríguez is with Structural Department, Alstom-Power Wind, Ecotecnia Energías Renovables SL, Roc Boronat, 78, 08005 Barcelona, Spain

J. Rodellar is with CoDAlab, Dep. de Matemàtica Aplicada III, Escola Tècnica Superior d'Enginyers de Camins, Canals i Ports de Barcelona (ETSECCPB), UPC, Jordi Girona Salgado, 1-3, 08034 Barcelona, Spain is that the assistance of such a force can help prevent large displacements of the base isolator, which could endanger the integrity of the scheme.

In this paper we firstly consider this kind of hybrid systems in which an active feedback control law is derived to be applied in parallel with a passive isolation device. The active feedback control law uses the restoring force supplied by the passive isolator as the main feedback signal.

Since semi-active controllers in hybrid base-isolation systems can achieve almost the same performance as an active base isolation system in protecting the safety of building against strong earthquakes [13], we also present the semi-active realization of the proposed active scheme. In this sense, the magnetorheological (MR) fluid dampers considered as semi-active devices- are represented using the normalized Bouc-Wen model [11]. Because the force generated in the MR dampers is dependent on the local responses of the structural system, the desired control force cannot always be produced by the devices. Only the control current or voltage can be directly controlled to increase or decrease the force produced by this devices. In this work, a new practical method [2], [3] is used to compute the command current of the MR dampers. The whole method is finally simulated by considering a three-dimensional smart base-isolated benchmark building [16].

The paper is structured as follows. Section II is dedicated to designing the force-derivative feedback control law and it is divided into two subsections: Subsection IIA presents the dynamic model of the base-isolated structure; Subsection II-B describes the desired control force which is based on a force-derivative feedback controller for hysteretic base-isolated structures. The semi-active realization of the proposed control scheme is developed in Section III. The inverse model that provides a suitable context to compute the command current of MR dampers analytically is described in Subsection III-A; meanwhile the algorithm for selecting the command signal is concisely stated in Subsection III-B. The smart base-isolated structure that serves as a benchmark problem for numerical testing is presented in Section IV together with some numerical simulations to analyze the performance of the proposed semi-active scheme. Final comments are given in Section V.

\section{FORCE-DERIVATIVE FEEDBACK CONTROL LAW DEVELOPMENT}

There exists a wide range of control algorithms that are applied to base-isolated buildings: clipped-optimal control [7], [12], [26]; maximum energy dissipation algorithms [14]; 
and modulated homogeneous friction algorithms, among others. Each of these controllers is able to reduce the structural response to some degree. From a structural point of view, a reasonable controller has to reduce the base displacement while decreases or slightly increases the accelerations. Li and $\mathrm{Ou}$ [13] showed that the active control forces in baseisolated structures have damping characteristics. In this study, an active force-derivative feedback controller will be applied in a semi-active way to the eighth-storied base-isolated benchmark building [16]. The control forces will be applied at the base through manipulation of the command current $i$ at the MR dampers.

\section{A. System description}

The system description considers a nonlinear base-isolated building structure as shown in Figure 1. More precisely, the control design is based on a dynamic model composed of two coupled subsystems, namely, the main structure or superstructure $\left(S_{r}\right)$ and the base isolation $\left(S_{c}\right)$ :

$$
\begin{aligned}
S_{r} & : \mathbf{M} \ddot{\mathbf{x}}=-\mathbf{M J} \ddot{x}_{g}-\mathbf{C} \dot{\mathbf{r}}-\mathbf{K r} \\
S_{c} & : m \ddot{x}+c \dot{x}+k x=c_{1} \dot{r}_{1}+k_{1} r_{1}-m \ddot{x}_{g}-\Phi+u
\end{aligned}
$$

where $\ddot{x}_{g}$ is the absolute ground acceleration, $\mathbf{x}=$ $\left[x_{1}, x_{2}, \ldots, x_{8}\right]^{\mathrm{T}} \in \mathbb{R}^{8}$ represents the horizontal displacements of each floor with respect to the ground. The mass, damping and stiffness of the $i$ th storey is denoted by $m_{i}, c_{i}$ and $k_{i}$, respectively, $\mathbf{r}=\left[r_{1}, \ldots, r_{8}\right]^{\mathrm{T}} \in \mathbb{R}^{8}$, represents the horizontal displacements of the $i$-th floor relative to the $(i-1)$-th floor. The base isolation is described as a single degree of freedom with horizontal displacement $x$. It is assumed to exhibit a linear behavior characterized by mass, damping and stiffness $m, c$ and $k$, respectively, plus a nonlinear behavior represented by a hysteretic restoring force $\Phi$. This restoring force can be supplied by some base isolation device. The matrices $\mathbf{M}, \mathbf{C}, \mathbf{K}$ and $\mathbf{J}$ of the structure have the following form:

$$
\begin{gathered}
\mathbf{M}=\operatorname{diag}\left(m_{1}, m_{2}, \ldots, m_{8}\right) \in \mathbb{R}^{8 \times 8} \\
\mathbf{J}=[1, \ldots, 1]^{\mathrm{T}} \in \mathbb{R}^{8} \\
\mathbf{C}=\left(c_{i j}\right) \in \mathbb{R}^{8 \times 8}, c_{i j}= \begin{cases}c_{i}, & i=j \\
-c_{i+1}, & j-i=1 \\
0, & \text { otherwise }\end{cases} \\
\mathbf{K}=\left(k_{i j}\right) \in \mathbb{R}^{8 \times 8}, k_{i j}= \begin{cases}k_{i}, & i=j \\
-k_{i+1}, & j-i=1 \\
0, & \text { otherwise }\end{cases}
\end{gathered}
$$

Finally, $u$ is the active control force supplied by an appropriate actuator.

The model in equations (1)-(2) is used to design an appropriate control law. The applicability and efficiency of the proposed controller will be then shown using a more realistic and complex model.
The equation of motion of the base (2) can be written in the form

$$
\begin{aligned}
S_{c}: m \ddot{x}+c \dot{x}+k x= & \underbrace{c_{1}\left(\dot{x}_{1}-\dot{x}\right)+k_{1}\left(x_{1}-x\right)}_{\delta\left[x, \dot{x}, x_{1}, \dot{x}_{1}\right]} \\
& \underbrace{-\Phi-m \ddot{x}_{g}}_{\Delta(t)}+u .
\end{aligned}
$$

It is well accepted that the movement of the superstructure $S_{r}$ is very close to the one of a rigid body due to the base isolation [18]. Then it is reasonable to assume that the motion of the first floor relative to the base will be very small. Therefore, it is also reasonable that the interaction force $\delta\left[x, \dot{x}, x_{1}, \dot{x}_{1}\right]$ will be small in comparison with the rest of the forces acting on the base [14]. Consequently, the following simplified equation of motion of the base can be used in the subsequent controller design:

$$
\tilde{S}_{c}: m \ddot{x}+c \dot{x}+k x=-\Phi-m \ddot{x}_{g}+u .
$$

\section{B. Force-derivative feedback controller}

Force-feedback controllers have received great attention in the active cable control of cable-stayed bridges [6], where the concept of integral force feedback is introduced, offering a sufficient increase of structure damping by suitable sensoractuator-pairs integrated between the stay-cables and the bridge deck. The static loads are compensated by hydraulic accumulators without permanent power supply. Moreover, force-feedback controllers have been seen in active vibration isolation [5], [19], where it is shown that the force feedback implementation benefits from alternating poles and zeros which allows a control law with guaranteed stability, making it very attractive when the payload to be isolated from the disturbance source is very flexible, such as in large space structures. At the same time, other applications have been seen in piezoelectric actuators for vibration control of civil structures used (decentralized) integral force feedback control [21], vehicle vibration control using MR dampers [23], and hybrid structural simulation [1] and [25].

Assuming that the earthquake disturbance is unknown but bounded, the following force-derivative feedback controller is proposed:

$$
u=-\zeta \dot{\Phi}
$$

where $\zeta$ is a positive real number. From an active control point of view, the force which has to be supplied by the actuator is based on the measured force which is currently supplied by the isolator device.

More precisely, the following assumptions are stated for system (3):

Assumption 1: The force $\Phi$ is described by the normalized version of the Bouc-Wen model [10]:

$$
\begin{aligned}
\Phi(t) & =\kappa_{\dot{x}} \dot{x}(t)+\kappa_{w} w(t), \\
\dot{w}(t) & =\rho\left(\dot{x}(t)-\sigma|\dot{x}(t)||w(t)|^{n-1} w(t)\right. \\
& \left.+(\sigma-1) \dot{x}(t)|w(t)|^{n}\right),
\end{aligned}
$$


where $\dot{x}$ is the velocity and $\kappa_{\dot{x}}, \kappa_{w}, \rho, \sigma$ and $n$ are the system parameters.

Assumption 2: The earthquake disturbance $-m \ddot{x}_{g}(t)$ is unknown but bounded; i.e., there exists a known constant $G$ such that $\left|\ddot{x}_{g}(t)\right| \leq G, \forall t \geq 0$.

Moreover, Theorem 1 in [10] guarantees the existence of a computable upper bound $\rho_{w}$ on the internal dynamic variable $w(t)$, i.e., $|w(t)| \leq \rho_{w}, \forall t \geq 0$, independently on the boundedness of $x(t)$.

The following theorem states the bounded-input boundedoutput stability of the proposed controller.

Theorem 1: Consider the nonlinear system in equation (3) and the force-derivative feedback control law

$$
u=-\zeta \dot{\Phi}
$$

under the assumption of the boundedness of the earthquake disturbance. Then, the closed-loop system in equations (3) and (7) is bounded-input bounded-output stable.

Proof. This proof is based on the boundedness of the earthquake disturbance $-m \ddot{x}_{g}$.

The closed-loop system in equations (3) and (7) yields

$m \ddot{x}+c \dot{x}+k x=-(\underbrace{\kappa_{\dot{x}} \dot{x}+\kappa_{w} w}_{\Phi})-m \ddot{x}_{g}-\zeta \underbrace{\left(\kappa_{\dot{x}} \ddot{x}+\kappa_{w} \dot{w}\right)}_{\dot{\Phi}}$.

Applying the Laplace transform to the equation of motion of the base, we obtain

$$
\begin{array}{r}
{\left[\left(m+\zeta \kappa_{\dot{x}}\right) s^{2}+\left(c+\kappa_{\dot{x}}\right) s+k\right] x(s)=} \\
-m \ddot{x}_{g}(s)-\left[\zeta \kappa_{w} s+\kappa_{w}\right] w(s),
\end{array}
$$

where $x(s), w(s)$ and $\ddot{x}_{g}(s)$ are the Laplace transform of the signals $x(t), w(t)$ and $\ddot{x}_{g}(t)$, respectively. The direct transfer function between the ground motion $\ddot{x}_{g}$, the hysteretic variable $w$, and the controlled base displacement $x$ is

$$
\begin{aligned}
x(s) & =\overbrace{\frac{-m}{\left(m+\zeta \kappa_{\dot{x}}\right) s^{2}+\left(c+\kappa_{\dot{x}}\right) s+k}}^{T_{g}(s)} \ddot{x}_{g}(s) \\
& -\underbrace{\frac{\zeta \kappa_{w} s+\kappa_{w}}{\left(m+\zeta \kappa_{\dot{x}}\right) s^{2}+\left(c+\kappa_{\dot{x}}\right) s+k}}_{T_{w}(s)} w(s) \\
& =T_{g}(s) \ddot{x}_{g}(s)-T_{w}(s) w(s)
\end{aligned}
$$

It can be shown, using the Nyquist stability criterion, that the transfer functions $T_{g}(s)$ and $T_{w}(s)$ are stable. Therefore, the boundedness of the input signals $\ddot{x}_{g}(t)$ and $w(t)$ guarantees the boundedness of the output signal $x(t)$, that is, the base displacement.

\section{SEMI-ACTIVE IMPLEMENTATION}

In this work, the active control force is not applied directly to the base through an active actuator. Contrarily, we use MR dampers to perform a semi-active realization. The first step used to design a semi-active control strategy is the selection of an active control law that makes the closed-loop system stable. In our case, this active control law is based on the derivative of the force which is currently supplied by the isolator device. The second step is the use of a semi-active MR damper, with force $F_{\mathrm{MR}_{p}}$, to try to follow the active desired force given by equation (7). That is, we use: (a) a passive isolator device described by equations (5)-(6) with fixed parameters and (b) a semi-active MR damper that tries, by updating the current $i$, to apply a force equal to the derivative of the force of the passive isolator device times a design parameter $-\zeta$. Even though this strategy can seem redundant, in the event of a fault, the system continues acting in a passive way. The next sections are concerned on how to compute the command current to carry out this objective.

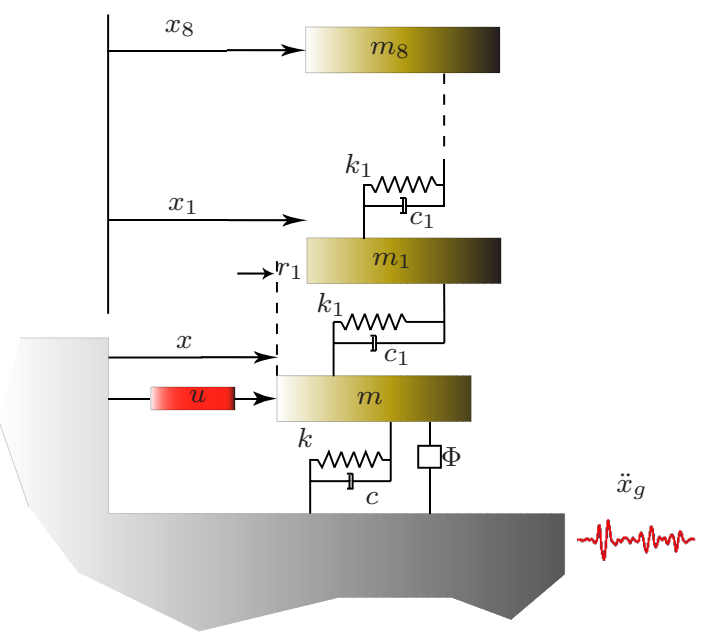

Fig. 1. Two degree-of-freedom (2DOF) model.

\section{A. The inverse model}

The inverse model will provide a suitable tool to compute the command current of MR dampers analytically. Consider the normalized form of the Bouc-Wen model for MR dampers (see the Appendix for further details):

$$
\begin{aligned}
F_{\mathrm{MR}}(t) & =\left[\kappa_{\dot{x}, a} i(t)+\kappa_{\dot{x}, b}\right] \dot{x}(t) \\
& +\left[\kappa_{w, a} i^{2}(t)+\kappa_{w, b} i(t)+\kappa_{w, c}\right] w(t),
\end{aligned}
$$

where $F_{\mathrm{MR}}(t)$ is the output force of the MR damper. The inverse model, that is, the computation of the current $i$ as a function of the velocity and force, is based on the following simplification:

(a) the internal dynamic variable $w(t)$, which is unmeasurable, is replaced by the sign of the velocity:

$$
w(t)=\operatorname{sgn}(\dot{x}) \in\{-1,1\} .
$$

We remark that, in the normalized version of the BoucWen model, the value of this internal dynamic variable lies within the range $[-1,1]$.

As a result of this simplification, the MR damper model is

$$
\begin{aligned}
F_{\mathrm{MR}}(t) & =\left[\kappa_{\dot{x}, a} i(t)+\kappa_{\dot{x}, b}\right] \dot{x}(t) \\
& +\left[\kappa_{w, a} i^{2}(t)+\kappa_{w, b} i(t)+\kappa_{w, c}\right] \operatorname{sgn}(\dot{x}) \\
& =\left[\kappa_{w, a} \operatorname{sgn}(\dot{x})\right] i^{2}(t)+\left[\kappa_{\dot{x}, a} \dot{x}(t)+\kappa_{w, b} \operatorname{sgn}(\dot{x})\right] i(t) \\
& +\left[\kappa_{\dot{x}, b} \dot{x}(t)+\kappa_{w, c} \operatorname{sgn}(\dot{x})\right]
\end{aligned}
$$


The current $i(t)$ can be found by solving the quadratic equation

$$
\begin{aligned}
\underbrace{\kappa_{w, a} \operatorname{sgn}(\dot{x})}_{a_{2}} & i^{2}(t)+\underbrace{\left[\kappa_{\dot{x}, a} \dot{x}(t)+\kappa_{w, b} \operatorname{sgn}(\dot{x})\right]}_{a_{1}} i(t) \\
& +\underbrace{\kappa_{\dot{x}, b} \dot{x}(t)+\kappa_{w, c} \operatorname{sgn}(\dot{x})-F_{\mathrm{MR}}(t)}_{a_{0}}=0 .
\end{aligned}
$$

Thereby, the final form of the inverse model will be:

$$
i\left(\dot{x}, F_{\mathrm{MR}}\right)=\frac{-a_{1} \pm \sqrt{a_{1}^{2}-4 a_{2} a_{0}}}{2 a_{2}}
$$

where

$$
\begin{aligned}
& a_{0}=\kappa_{\dot{x}, b} \dot{x}(t)+\kappa_{w, c} \operatorname{sgn}(\dot{x})-F_{\mathrm{MR}}(t) \\
& a_{1}=\left[\kappa_{\dot{x}, a} \dot{x}(t)+\kappa_{w, b} \operatorname{sgn}(\dot{x})\right] \\
& a_{2}=\kappa_{w, a} \operatorname{sgn}(\dot{x})
\end{aligned}
$$

\section{$B$. The selection of the command current $i$}

It is well known that the force generated by the MR damper cannot be commanded; only the voltage $v$ or the current $i$ applied to the MR damper can be directly changed [7]. In the clipped-optimal control algorithm [7], the command voltage takes the values zero or the maximum, according to

$$
v=V_{\max } H\left\{\left(f_{\mathrm{d}}-F_{\mathrm{MR}}\right) F_{\mathrm{MR}}\right\},
$$

where $V_{\max }$ is the maximum voltage to the current driver associated with saturation of the magnetic field in the MR damper, $H(\cdot)$ is the Heaviside step function, $f_{\mathrm{d}}$ is the desired control force and $F_{\mathrm{MR}}$ is the measured force of the MR damper. In some situations, when the dominant frequencies of the system under control are low, large changes in the forces applied to the structure may result in high local acceleration [26]. In this sense, a modification to the original clipped-optimal control algorithm in which the control voltage can be any value between zero and a $V_{\max }$, was proposed in [26], where the control voltage is determined using a linear relationship between the applied voltage and the maximum force of MR damper. A similar approach can be found in [8], where a force-feedback control scheme is employed to overcome the difficulty of commanding the MR damper to produce an arbitrary force. In this paper we consider the same idea of changing the voltage but changing the current according to the inverse model in equation (8). More precisely, to induce the MR damper to generate approximately the desired control force $f_{\mathrm{d}}$, the algorithm for selecting the command signal can be concisely stated as

$$
i=\frac{-a_{1} \pm \sqrt{a_{1}^{2}-4 a_{2} a_{0}}}{2 a_{2}}
$$

where

$$
\begin{aligned}
& a_{0}=\kappa_{\dot{x}, b} \dot{x}(t)+\kappa_{w, c} \operatorname{sgn}(\dot{x})-f_{\mathrm{d}}(t) \\
& a_{1}=\left[\kappa_{\dot{x}, a} \dot{x}(t)+\kappa_{w, b} \operatorname{sgn}(\dot{x})\right] \\
& a_{2}=\kappa_{w, a} \operatorname{sgn}(\dot{x})
\end{aligned}
$$

and $f_{\mathrm{d}}$ is computed according to

$$
f_{\mathrm{d}}=-\zeta \dot{\Phi} .
$$

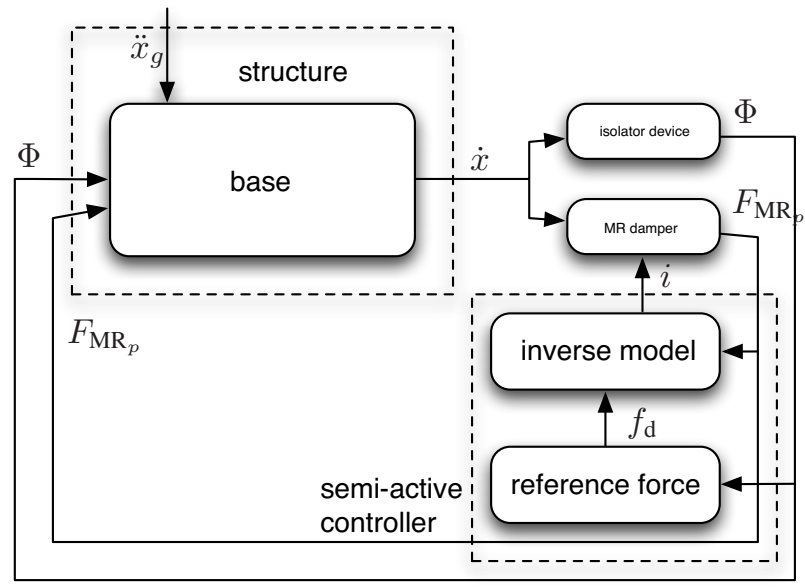

Fig. 2. Block diagram of the semi-active control system for a single MR damper.

Both equations (10)-(11) define a semi-active controller. Figure 2 illustrates the corresponding closed-loop system.

Based on this desired force, the corresponding command current that has to be applied to the damper will be calculated according to equation (10). Let $i_{\alpha}$ and $i_{\beta}$ be the two roots of this equation and let $\left[0, i_{\max }\right]$ be the range of admissible current. The applied current $i_{a}$ will finally be

$$
i_{a}= \begin{cases}\max \left\{i_{\alpha}, i_{\beta}\right\}, & i_{\alpha}, i_{\beta} \in\left[0, i_{\max }\right] \\ \min \left\{i_{\alpha}, i_{\beta}\right\}, & \min \left\{i_{\alpha}, i_{\beta}\right\} \in\left[0, i_{\max }\right], \\ & \max \left\{i_{\alpha}, i_{\beta}\right\} \notin\left[0, i_{\max }\right] \\ i_{\max }, & i_{\alpha}, i_{\beta}>0, \min \left\{i_{\alpha}, i_{\beta}\right\} \notin\left[0, i_{\max }\right] \\ \max \left\{i_{\alpha}, i_{\beta}\right\}, & \min \left\{i_{\alpha}, i_{\beta}\right\}<0, \\ & \max \left\{i_{\alpha}, i_{\beta}\right\} \in\left[0, i_{\max }\right] \\ i_{\max }, & \min \left\{i_{\alpha}, i_{\beta}\right\}<0, \\ & \max \left\{i_{\alpha}, i_{\beta}\right\} \geq i_{\max } \\ 0, & \max \left\{i_{\alpha}, i_{\beta}\right\}<0 \\ \Re\left(i_{\alpha}\right), & i_{\alpha} \in \mathbb{C} \backslash \mathbb{R}, 0 \leq \Re\left(i_{\alpha}\right) \leq i_{\max } \\ 0, & i_{\alpha} \in \mathbb{C} \backslash \mathbb{R}, \Re\left(i_{\alpha}\right)<0 \\ i_{\max }, & i_{\alpha} \in \mathbb{C} \backslash \mathbb{R}, \Re\left(i_{\alpha}\right)>i_{\max }\end{cases}
$$

In the implementation of this formula, the values are truncated between zero and $i_{\max }$ when the current does not belong to the range of admissible values.

When the roots $i_{\alpha}$ and $i_{\beta}$ of the equation $a_{2} i^{2}(t)+a_{1} i(t)+$ $a_{0}=0$ are real and belong to the range of admissible values, it is clear that that both equations $a_{2} i_{\alpha}^{2}+a_{1} i_{\alpha}+a_{0}=0$ and $a_{2} i_{\beta}^{2}+a_{1} i_{\beta}+a_{0}=0$ are satisfied. Defining $f(i)=a_{2} i^{2}(t)+$ $a_{1} i(t)+a_{0}$, we can conclude that $f\left(i_{\alpha}\right)=0$ and $f\left(i_{\beta}\right)=0$ being $i_{\alpha}, i_{\beta} \in \mathbb{R}$. We can equivalently say that $i_{\alpha}$ and $i_{\beta}$ are the unique two values that minimizes the expression $|f(i)|$. When the roots $i_{\alpha}, i_{\beta} \in \mathbb{C} \backslash \mathbb{R}$ are complex conjugates, we choose the applied current $i_{a}$ based on this idea: we solve the problem $\min _{i \in \mathbb{R}}|f(i)|$. Considering that $f(i)$ is a parabola with no real roots, the value that minimizes this expression is its vertex

$$
i_{a}=\frac{-a_{1}}{2 a_{2}}=\Re\left(\frac{-a_{1} \pm \sqrt{a_{1}^{2}-4 a_{2} a_{0}}}{2 a_{2}}\right)=\Re\left(i_{\alpha}\right),
$$




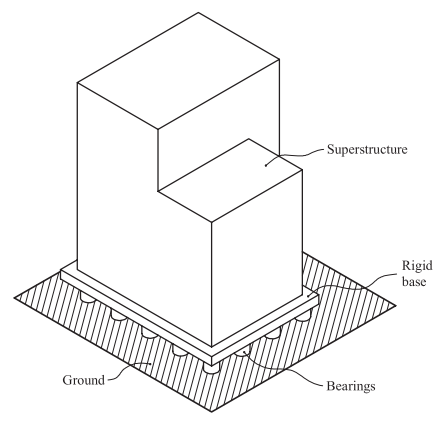

Fig. 3. A representative figure of the benchmark structure.

that is, the real part of the complex conjugate roots.

This process helps the damping force generated by the MR dampers become more closer to the desired control force, $f_{\mathrm{d}}$.

\section{NUMERICAL RESULTS}

The performance of the semi-active control algorithm presented in Section III is now evaluated through numerical simulation using the smart base-isolated benchmark building. The smart base-isolated benchmark building [16] is employed as an interesting and more realistic example to further investigate the effectiveness of the proposed design approach. This base-isolated building is an eight-storey frame building, similar to existing building in Los Angeles, California. The results are also compared with the clipped-optimal control algorithm in equation (9) [7] and also with two limit cases: passive off and passive on, that corresponds to the cases of zero current applied to the damper and maximum current applied to the damper. The evaluation is reported in terms of the performance indices described in Table I. The controlled benchmark structure is simulated for seven earthquake ground accelerations defined in the benchmark problem (Newhall, Sylmar, El Centro, Rinaldi, Kobe, Ji$\mathrm{Ji}$ and Erzinkan). The performance indices larger than 1 indicate that the response of the controlled structure is bigger than that of the uncontrolled structure. Table II shows the average evaluation criteria for all 14 cases (the seven prescribed earthquakes in two orthogonal directions). The performance indices larger than one in Table II are underlined. Figure 4 show the time history response of the base-isolated building under the Erzinkan earthquake for different control cases. It is clear in Figure 4 that the proposed semi-active controller reduces the base displacements in $75 \%$ from the passive-off case.

\section{CONCLUDING REMARKS}

In this paper we have considered a hybrid system in which an active feedback control law has been derived to be applied in parallel with a passive isolation device. The active control uses the restoring force supplied by the passive isolator as the main feedback signal. A set of numerical simulations have been performed when the force is supplied in a semi-active way to validate and discuss the efficiency of the approach in a more realistic scenario. With respect to the implementation issues, a new practical method has been defined to compute

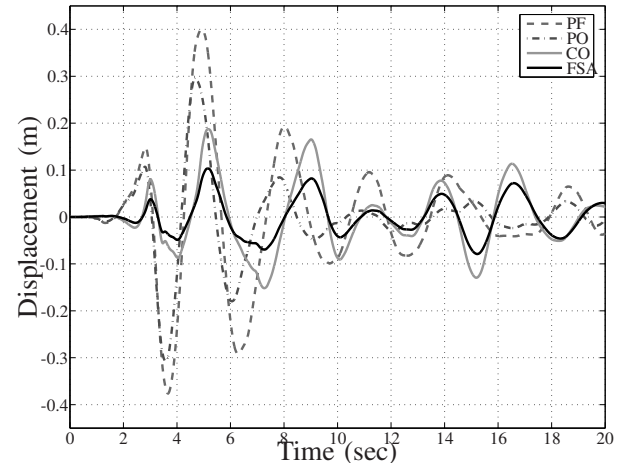

Fig. 4. Response time history of the building subjected to Erzinkan earthquake (FP-y and FN- $x$ ) base displacement at the center of mass (2000kN MR damper) [PO=passive on, $\mathrm{PF}=$ passive off, $\mathrm{CO}=$ clipped optimal, FSA=proposed].

TABLE I

Performance Indices, Where, $i=$ ISOLATOR NUMBer, $1, \ldots, n_{\mathrm{MR}}$; $k=$ DeVICE NUMBER, $1, \ldots, N_{d} ; f=$ FLOOR NUMBER, $1, \ldots, N_{f} ; q=$ EARTHQUAKE NUMBER, $1, \ldots, 7 ; t=$ TIME, $0 \leq t \leq T_{q} ;\langle\cdot\rangle=$ INNER PRODUCT; $\|\cdot\|=$ VECTOR MAGNITUDE INCORPORATING NS AND EW COMPONENTS.

\begin{tabular}{cc}
\hline Peak base shear & Peak structure shear \\
$J_{1}=\frac{\max _{t}\left\|V_{0}(t, q)\right\|}{\max _{t}\left\|\hat{V}_{0}(t, q)\right\|}$ & $J_{2}=\frac{\max _{t}\left\|V_{1}(t, q)\right\|}{\max _{t}\left\|\hat{V}_{1}(t, q)\right\|}$ \\
\hline Peak base displacement & Peak inter-storey drift \\
$J_{3}=\frac{\max _{t, i}\left\|d_{i}(t, q)\right\|}{\max _{t, i}\left\|\hat{d}_{i}(t, q)\right\|}$ & $J_{4}=\frac{\max _{t, f}\left\|d_{f}(t, q)\right\|}{\max _{t, f}\left\|\hat{d}_{f}(t, q)\right\|}$ \\
\hline Peak Floor acceleration & Peak control force \\
$J_{5}=\frac{\max _{t, f}\left\|a_{f}(t, q)\right\|}{\max _{t, f}\left\|\hat{a}_{f}(t, q)\right\|}$ & $J_{6}=\frac{\max _{t}\left\|\sum_{k} F_{k}(t, q)\right\|}{\max _{t}\left\|V_{0}(t, q)\right\|}$ \\
\hline RMS base displacement & RMS floor acceleration \\
$J_{7}=\frac{\max _{i}\left\|\sigma_{d}(t, q)\right\|}{\max _{i}\left\|\sigma_{\hat{d}}(t, q)\right\|}$ & $J_{8}=\frac{\max _{f}\left\|\sigma_{a}(t, q)\right\|}{\max _{f}\left\|\sigma_{\hat{a}}(t, q)\right\|}$ \\
\hline Energy dissipated by MR damper \\
$J_{9}=\frac{\sum_{k}\left[\int_{0}^{T} F_{q} F_{k}(t, q) v_{k}(t, q) d t\right]}{\int_{0}^{T}\left\langle V_{0}(t, q) \dot{U}_{g}(t, q) d t\right\rangle}$ \\
\hline
\end{tabular}

TABLE II

AVERAGE EVALUATION CRITERIA FOR DIFFERENT CONTROLLERS USING 2000 KN MR DAMPERS FOR EARTHQUAKE EXCITATIONS IN BOTH DIRECTIONS

\begin{tabular}{ccccc}
\hline Indices & F. Feedback & Clipped & Passive-On & Passive-Off \\
\hline$J_{1}$ & 0.83 & 0.82 & 0.81 & 0.83 \\
$J_{2}$ & 0.83 & 0.81 & 0.82 & 0.86 \\
$J_{3}$ & 0.59 & 0.54 & 0.48 & 0.41 \\
$J_{4}$ & 0.85 & 0.87 & 0.94 & $\underline{1.09}$ \\
$J_{5}$ & 0.92 & $\underline{1.00}$ & $\underline{1.15}$ & $\underline{1.40}$ \\
$J_{6}$ & 0.29 & 0.33 & 0.45 & 0.14 \\
$J_{7}$ & 0.44 & 0.36 & 0.28 & 0.23 \\
$J_{8}$ & 0.59 & 0.57 & 0.59 & 0.58 \\
$J_{9}$ & 0.73 & 0.79 & 0.85 & 0.88 \\
\hline
\end{tabular}

the command voltage of the dampers according to the desired control force. The whole method is simulated by considering 
a three-dimensional smart base-isolated benchmark building which is used by the structural control community as a stateof-the-art model for numerical experiments of seismic control attenuation. The performance indices demonstrate that the proposed semi-active method can effectively suppress structural vibration caused by earthquake loading and can provide a desirable effect of structural performance.

\section{APPENDIX}

\section{THE MAGNETORHEOLOGICAL DAMPER MODEL}

The normalized version of the Bouc-Wen model [10] is an equivalent representation of the original Bouc-Wen model [24]. For MR dampers in shear mode it takes the form:

$$
\begin{aligned}
F_{\mathrm{MR}}(t) & =\kappa_{\dot{x}} \dot{x}(t)+\kappa_{w} w(t), \\
\dot{w}(t) & =\rho\left(\dot{x}(t)-\sigma|\dot{x}(t)||w(t)|^{n-1} w(t)\right. \\
& \left.+(\sigma-1) \dot{x}(t)|w(t)|^{n}\right),
\end{aligned}
$$

where $F_{\mathrm{MR}}(t)$ is the output force of the MR damper, and $\dot{x}(t)$ is the velocity. The system parameters, which are currentdependent, are $\kappa_{\dot{x}}(i)>0, \kappa_{w}(i)>0, \rho(i)>0, \sigma(i)>1 / 2$, and $n(i) \geq 1$. These parameters control the shape of the hysteresis loop and their meaning can be found in [11]. The state variable $w(t)$ has not a physical meaning so that it is not accessible to measurements.

The MR damper model in equations (14)-(15), which is based on the normalized Bouc-Wen model in equations (12)-(13), was obtained from a model validation of a largescale magnetorheological damper at Kinki University (Osaka, Japan) using both constant and varying current along with a varying displacement signal [20]. This normalized Bouc-Wen model depicts its current dependent parameters in equations (17)-(19):

$$
\begin{aligned}
F_{\mathrm{MR}}(t) & =\left[\kappa_{\dot{x}, a} i(t)+\kappa_{\dot{x}, b}\right] \dot{x}(t) \\
+ & {\left[\kappa_{w, a} i^{2}(t)+\kappa_{w, b} i(t)+\kappa_{w, c}\right] w(t) } \\
\dot{w}(t)=\left[\rho_{a} i^{2}(t)+\rho_{b} i(t)+\rho_{c}\right] \cdot & \\
\cdot & \left(\dot{x}(t)-\sigma|\dot{x}(t)||w(t)|^{n-1} w(t)\right. \\
+ & \left.(\sigma-1) \dot{x}(t)|w(t)|^{n}\right) \\
w(0) & =\frac{F(0)-\left[\kappa_{\dot{x}, a} i(0)+\kappa_{\dot{x}, b}\right] \dot{x}(0)}{\left[\kappa_{w, a} i^{2}(0)+\kappa_{w, b} i(0)+\kappa_{w, c}\right]} \\
\kappa_{\dot{x}}(i)= & \kappa_{\dot{x}, a} i+\kappa_{\dot{x}, b}=328.47 i+35.14 \mathrm{~N} / \mathrm{mm} \\
\kappa_{w}(i)= & \kappa_{w, a} i^{2}+\kappa_{w, b} i+\kappa_{w, c} \\
= & -136910 i^{2}+62530 i+600 \mathrm{~N} \\
\rho(i) & =\rho_{a} i^{2}+\rho_{b} i+\rho_{c} \\
= & 12.25 i^{2}-3.8 i+0.93 \mathrm{~mm}^{-1}
\end{aligned}
$$

The parameters $n$ and $\sigma$ barely show dependence on the current; therefore, constant average values are considered, $n=1.63$ and $\sigma=1.85$.

\section{REFERENCES}

[1] Ahmadizadeh M, Mosqueda G, Reinhorn AM. Compensation of actuator delay and dynamics for real-time hybrid structural simulation. Earthquake Engineering \& Structural Dynamics 2008; 37:21-42.
[2] Bahar A, Pozo A, Acho L, Rodellar J, Barbat A. Parameter identification of large-scale magnetorheological dampers in a benchmark building. Computers \& Structures 2010; 88(3-4):198-206.

[3] Bahar A, Pozo A, Acho L, Rodellar J, Barbat A. Hierarchical semiactive control of base-isolated structures using a new inverse model of magnetorheological dampers. Computers \& Structures, 2010; 88(78):483-496.

[4] Barbat A, Rodellar J, Ryan E, Molinares N. Active control of nonlinear base-isolated buildings. ASCE Journal of Engineering Mechanics, 1995; 121(6):676-684.

[5] Benassi L, Elliott SJ, Gardonio P. Active vibration isolation using an inertial actuator with local force feedback control. Journal of Sound and Vibration 2004; 276:157-179.

[6] Bossens F, Preumont A. Active tendon control of cable-stayed bridges: a large-scale demonstration. Earthquake Engineering \& Structural Dynamics 2001; 30:961-979.

[7] Dyke SJ, Spencer BF, Sain MK, Carlson JD. Modeling and control of magnetorheological dampers for seismic response reduction. Smart Materials and Structures 1996; 5(5):565-575.

[8] Gu ZQ, Oyadiji SO. Application of MR damper in structural control using ANFIS method. Computers \& Structures 2008; 86(3-5):427436.

[9] Housner GW, Bergman LA, Caughey TK, Chassiakos AG, Claus RO, Masri SF, Skelton RE, Soong TT, Spencer BF, Yao JTP. Structural control: past, present, and future. Journal of Engineering Mechanics (ASCE) 1997; 123(9):897-971.

[10] Ikhouane F, Mañosa V, Rodellar J. Adaptive control of a hysteretic structural system. Automatica 2005; 41(2):225-231.

[11] Ikhouane F, Rodellar J. Systems with Hysteresis: Analysis, Identification and Control using the Bouc-Wen Model. Wiley: Chichester, 2007.

[12] Jung HJ, Choi KM, Spencer BF, Lee IW. Application of some semiactive control algorithms to a smart base-isolated building employing MR dampers. Structural Control and Health Monitoring 2006; 13(23): 693-704.

[13] Li H, Ou J. A design approach for semi-active and smart baseisolated buildings. Structural Control and Health Monitoring 2006; 13(2-3):660-681.

[14] Luo N, Rodellar J, Vehí J, De la Sen M. Composite semiactive control of a class of seismically excited structures . Journal of the Franklin Institute 2001; 13(2-3):660-681.

[15] Naeim F, Kelly JM. Design of Seismic Isolated Structures: From Theory to Practice. John Wiley \& Sons, 1999.

[16] Narasimhan S, Nagarajaiah S, Johnson EA, Gavin HP. Smart baseisolated benchmark building. Part I: problem definition. Structural Control and Health Monitoring 2006; 13(2-3):573-588.

[17] Pozo F, Acho L, Rodellar J. Hyperbolic control for vibration mitigation of a base-isolated benchmark structure. Structural Control and Health Monitoring 2009; 16(7-8):766-783, doi: 10.1002/stc.339.

[18] Pozo F, Montserrat PM, Rodellar J, Acho L. Robust active control of hysteretic base-isolated structures: Application to the benchmark smart base-isolated building. Structural Control and Health Monitoring 2008; 15(5):720-736.

[19] Preumont A, François A, Bossens F, Abu-Hanieh A. Force feedback versus acceleration feedback in active vibration isolation. Journal of Sound and Vibration 2002; 257(4):605-613.

[20] Rodríguez A, Iwata N, Ikhouane F, Rodellar J. Model identification of a large-scale magnetorheological fluid damper. Smart Materials and Structures 2009; 18:1-12.

[21] Songa G, Sethi V, Lic HN. Vibration control of civil structures using piezoceramic smart materials: A review. Engineering Structures 2006; 28(11):1513-1524.

[22] Soong TT, Dargush GF. Passive Energy Dissipation Systems in Structural Engineering. John Wiley and Sons: UK, 1997.

[23] Wang ER, Ma XQ, Rakheja S, Su CY. Semi-active control of vehicle vibration with MR dampers. Proceedings of the 42nd IEEE Conference on Decision and Control 2003; Maui, Hawaii, USA.

[24] Wen YK. Method of random vibration of hysteretic systems. ASCE Journal Engineering Mechanics 1976; 102:249-263.

[25] Wu B, Wang Q, Shing PB, Ou J. Equivalent force control method for generalized real-time substructure testing with implicit integration. Earthquake Engineering \& Structural Dynamics 2007; 36(9):11271149.

[26] Yoshida O, Dyke SJ. Seismic control of a nonlinear benchmark building using smart dampers. Journal of Engineering Mechanics 2004; 130(4):386-392. 